\title{
ACTIVATING THE ROLE OF THE ARCHITECTURAL EDUCATION AS A CATALYST TO PRESERVE THE RESPONSIVENESS OF THE URBAN HERITAGE JEDDAH HISTORICAL AREA
}

\section{Nabil Al Kassar PhD.}

Assistant Professor, Department of Architecture

Faculty of Environmental Design, King Abdul Aziz University

Jeddah-Saudi Arabia

(Received April 18, 2010 Accepted May 15, 2010.)

The goal of this research is to examine a methodology in Architectural Education to test the responsiveness of the Urban Heritage environment. The problem was that the historical area in old Jeddah city was not properly covered in both the urban or the architectural academic studies, some efforts were done but they were not enough to fill the gap,. Furthermore there was a full absence of any image that should be given to the students to preserve or develop the local historical precedent. The author tried through an organized study to raise the awareness of his students about this part of knowledge. A tool was designed to help students understanding the local historical environment with a sound architectural and urban study that can reveal its urban and architectural problems and then in turn suggest specific and clear solutions that can be applied in the real world

The research sample was designed in a multi level system that can be examined practically in the specified location. Seven levels were examined permeability, variety, legibility, robustness, visual appropriateness, richness and personalization. All data were collected through site surveys, the urban reports that were saved by the Faculty of Environmental Design, and other data from previous workshops held by Jeddah Municipality about historical Jeddah. Some questionnaires were designed as a demand for some qualities examined in the study.

The research proves the success of the examined methodology to specify the key problems of the environment and then in turn to suggest and recommend the proper solutions for them.

KEYWORDS: permeability, variety, legibility, robustness, visual appropriateness, richness, personalization and historical Jeddah 


\section{تفعيل دور التعليم المعماري كأداة للحفاظ علي حيوية البيئة التقليدية

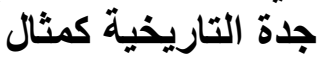

\section{د. نبيل عبد الرحمن الكسار \\ الأستاذ المساعد بقسم العمارة \\ كلية تصاميم البيئة \\ جامعة الملك عبد العزيز - جدة المانة}

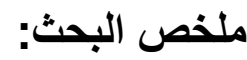

يهدف البحث إلي اختبار منهجية دراسية في التعليم المعماري يمكن أن تستخدم كوسيلة تحدد مدي حيوية البيئة

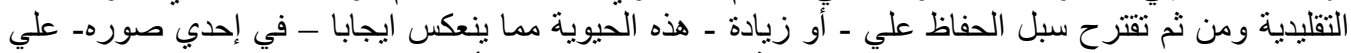

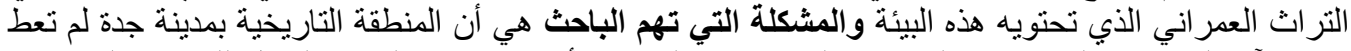

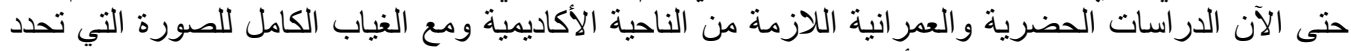

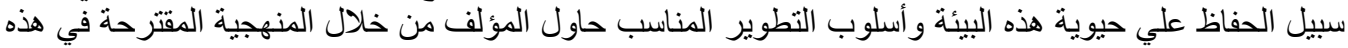

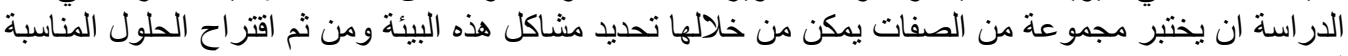

صممت عينة البحث في صورة مجمو عة من المستويات (هي النفاذية و التتوع و الوضوح و وحيوية المكان و الملائمة

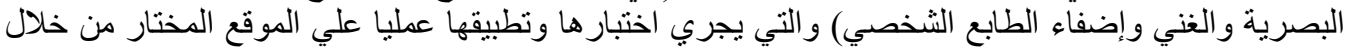

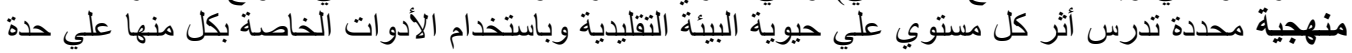

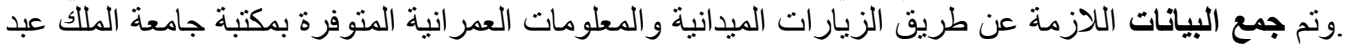

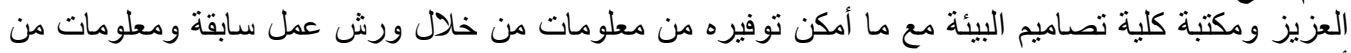

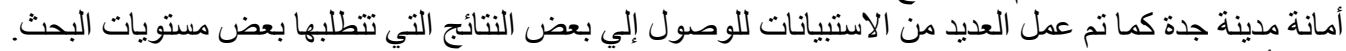

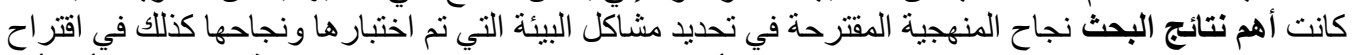

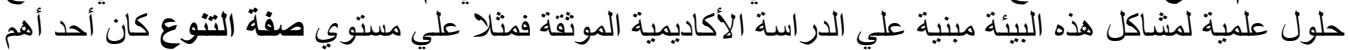

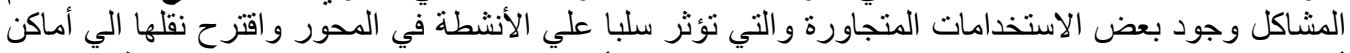

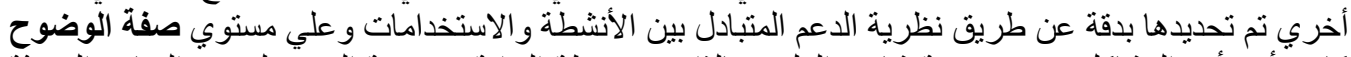

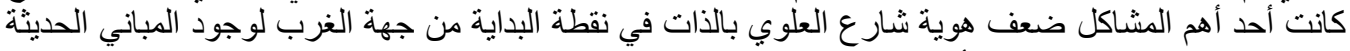

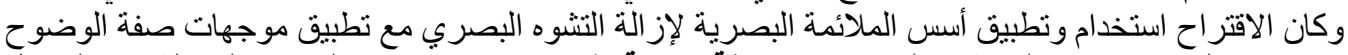

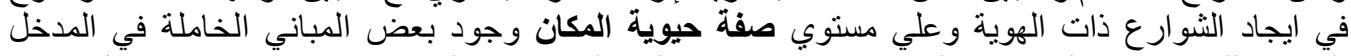

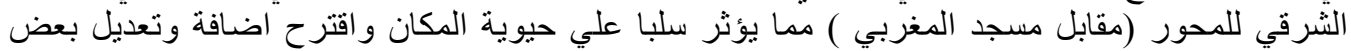
الاستخدامات للحصول علي و اجهات نشطة لهذه المباني و هكذا في بقية الصفات الصنات

الكلمات المفتاحية: البيئة التقليديةـ جدة التاريخية ـ النفاذية ـ التنوع- الوضوح - حيوية المكان ـ الغني - ثراء

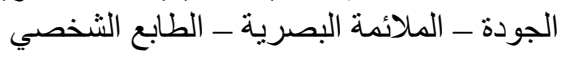

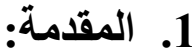

كيف يمكن أن نحكم علي بيئة ما بأنها حية وغير ميتة؟ و الجواب يكمن في مدي استجابة هذه البيئة لتحقيق رغبات

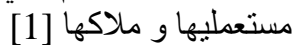

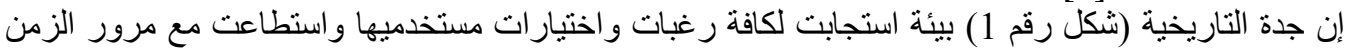

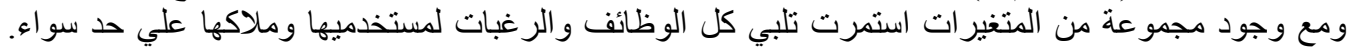

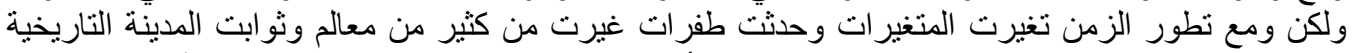

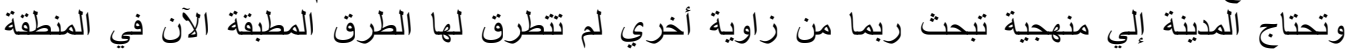
التاريخية.بمدينة جدة. 

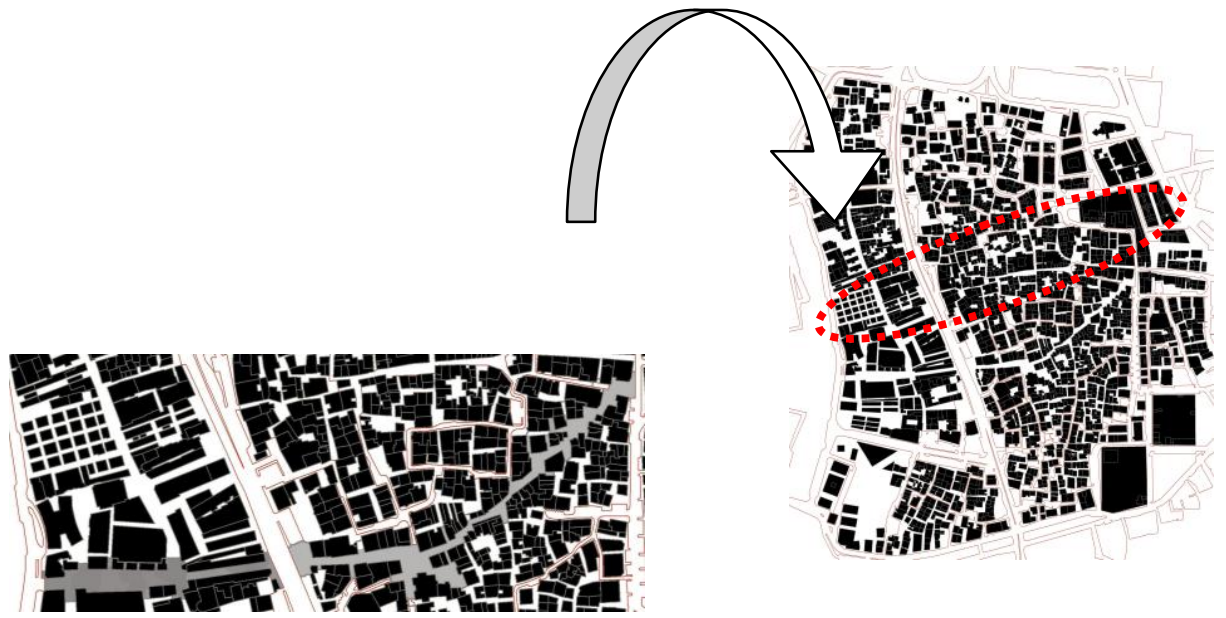

شكل رقم 1: موقع منطقة الدر اسة ـ المنطقة التاريخية بمدينة جدة ـ محور العلوي [2]

1.1 موضوع البحث: إن تصميم الأماكن يؤثر على اختيار ات الناس - و هذه حقيقةـ ونحن في هذا البحث حاولنا

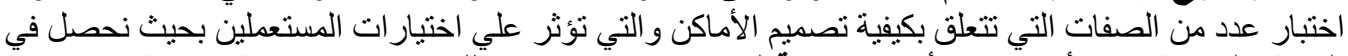

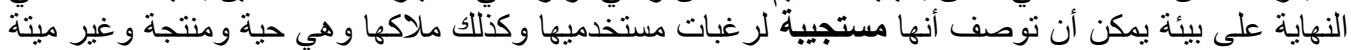
أو معطلة. 2.1 شأن هذا البحث بين الأبحاث التي ألفت في ذات الموضوع: هذا البحث ربما يكون الأول من نوعه الذي

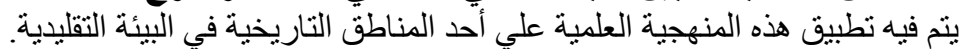

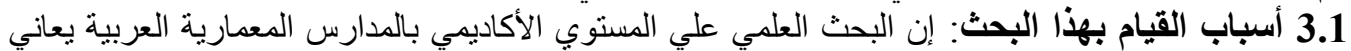

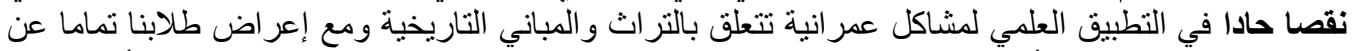

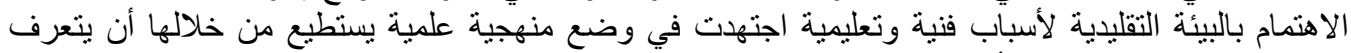

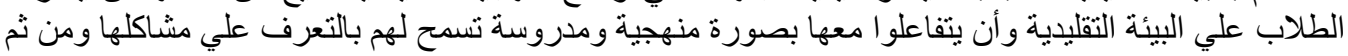

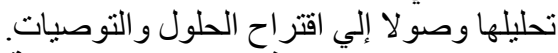

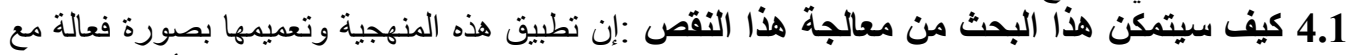

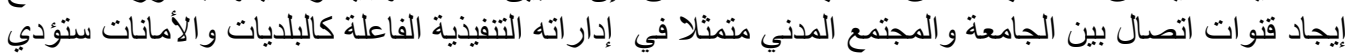

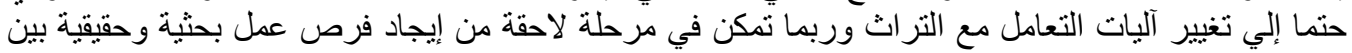

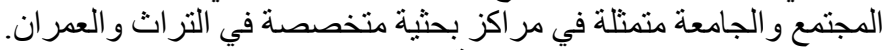

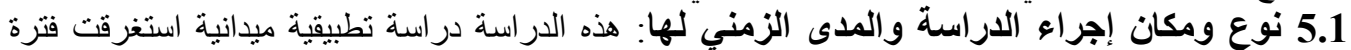

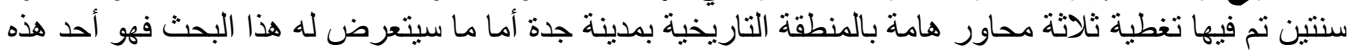

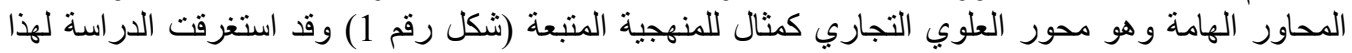
المحور فترة فصلين در اسيين في العام 1429 هـ لهـ - 2008 م.

6.1 الجهات التي ستنتفع بهذا البحث: إن المنهجية المتبعة في هذه الدراسة تتيح للجامعات والأقسام

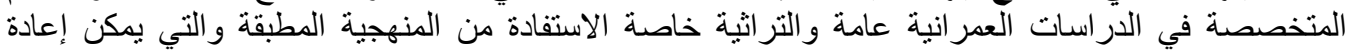

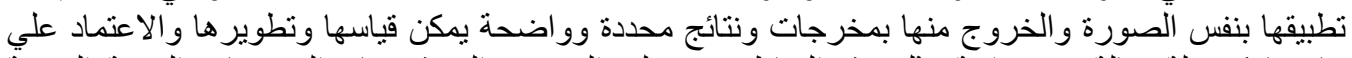

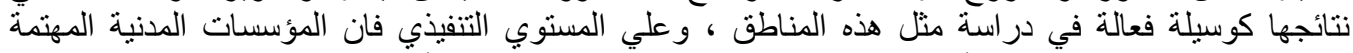

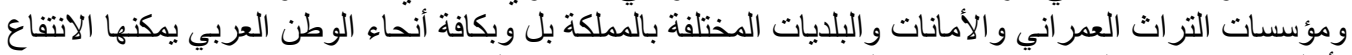

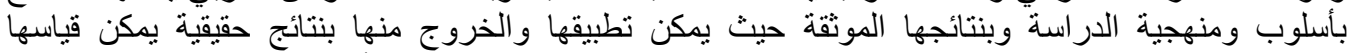
و الاعتماد عليها كوسيلة للتنخل في معالجة وتطوير البيئات التقليدية في مختلف أنحاء هذا الوطن الغني بتراثئ 


\section{1. الإطار النظري للاراسة :}

يفترض البحث ان هناك شبكة من العلاقات بين الصفات التي يختبرها البحث وبين حيوية البيئة التقليدية وأن

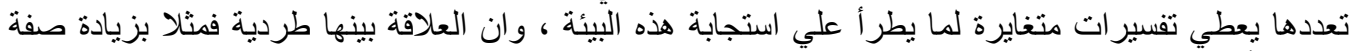

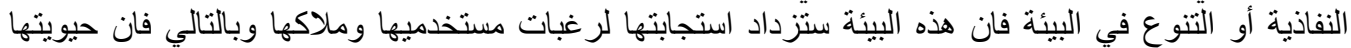

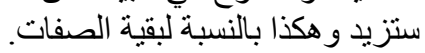
2.

لما كان الهدف الرئيسي للبحث هو زيادة حيوية البيئة التقليدية في منطقة جدة التاريخية من خلال در اسة مجمو عة دئة

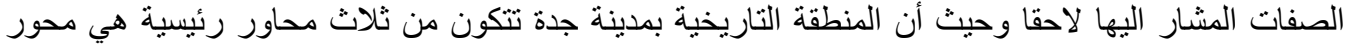

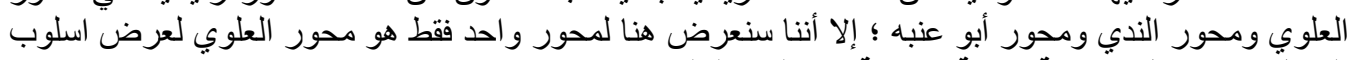

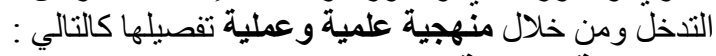

المنهجية العلمية خلية

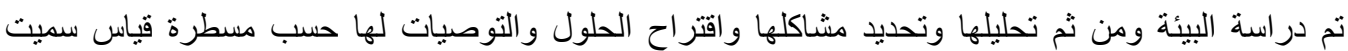

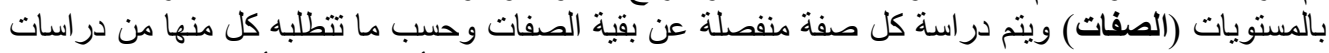

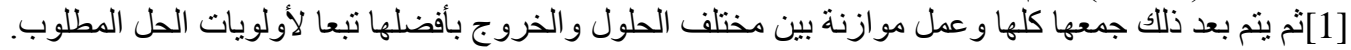

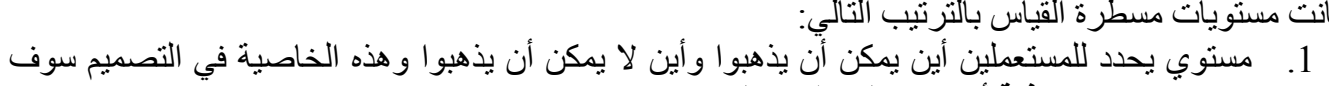

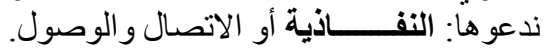

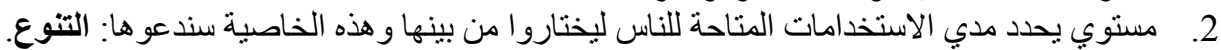

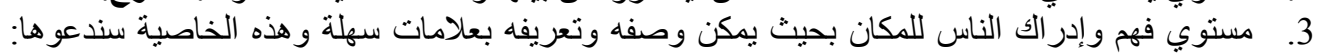
4. مستوي يحدد إلي أي مدي أو درجة يمكن للناس استخدام نفس المكان بعدة طرق ولعدة وظائف وهذه

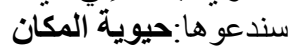
5. مستوي يحدد تفاصيل المكان البصرية والتي تؤنثر علي إدران الك الناس للاستعمالات المختلفة لهذا المكان و هذه سندعو ها الملائمة البصرية. 6. مستوي يحدد التجربة الحسية للمستعملين وأثر ها علي الختيار ات الناس وهذه سندعوها: الغتي أو ثراء الجودة مستوي يحدد إلي أي مدي يمكن أن يضع المستخدم طابعة الشخصي في المكان و هذه الخاصية سندعو ها: .7

اشتملت كُل صفة علي ثلاثة مستّويات : المفهوم و الإطار النظري لدراسة هذه الصفة ومضامين التصميم :

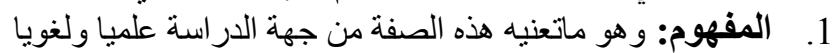

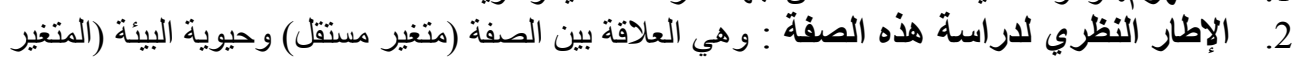

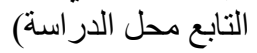
3. مضامين التصميم: الطريقة التي اتبعت لدراسة وتطبيق كل صفة علي حدة حسب متطلباتها

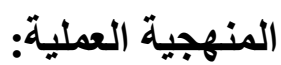
اشتملت هذه المنهجية علي الطريقة التي يتم بها تتفيذ الدر اسة العلمية للبيئة وهي كالتالي:

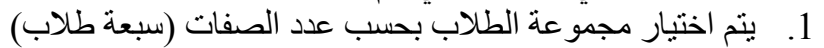

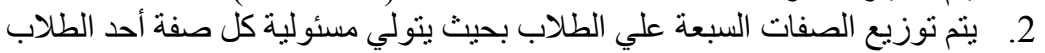

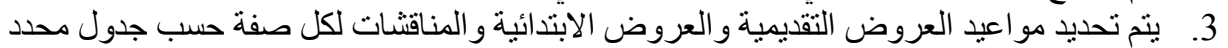

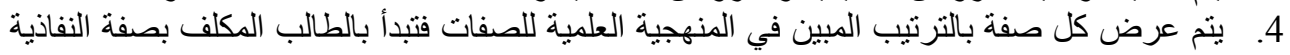

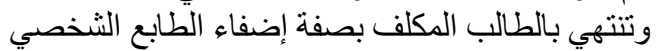

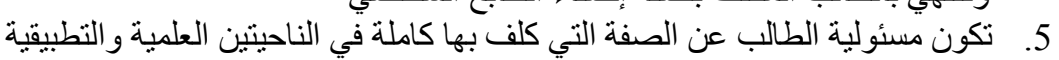

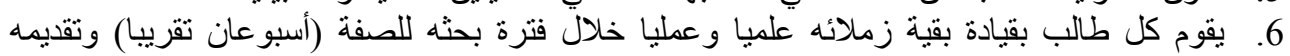

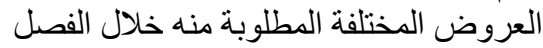


7. يشترك جميع الطلاب في تطبيق جميع الصفات ولكن مع تبادل الأدوار بحيث يتعلم الجميع من الجميع

$$
\text { 8. }
$$

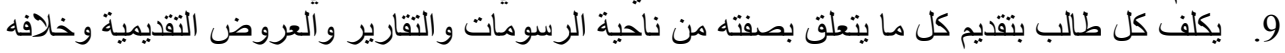

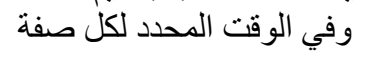

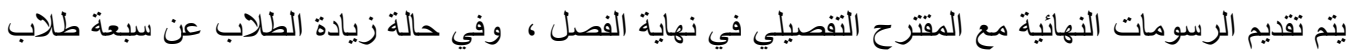

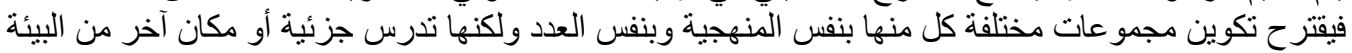

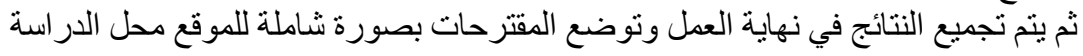

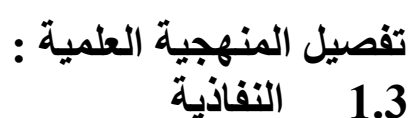

المفهوم : الأماكن سهلة الاتصال و الوصول للناس والتي توفر للمستخدمين خيار ات الوصول من مكان إلى آخر

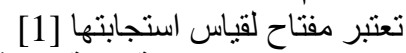

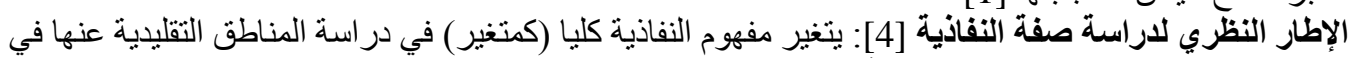

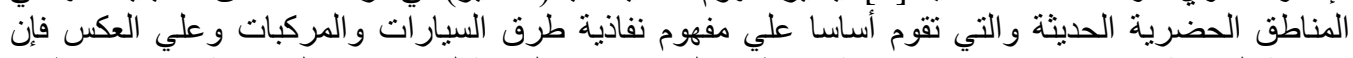

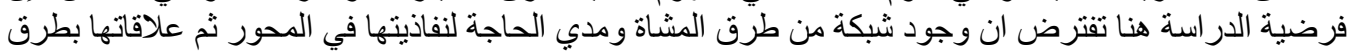

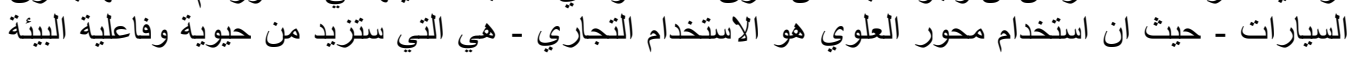
التجارية بالمنطقة التاريخية

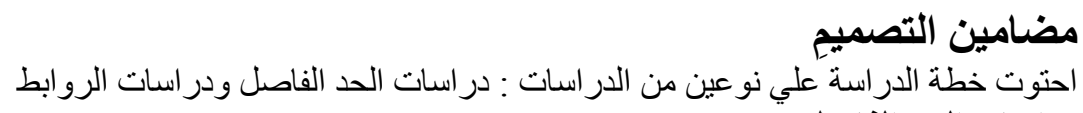

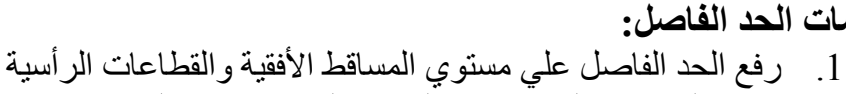

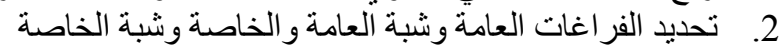

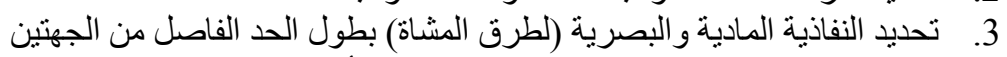

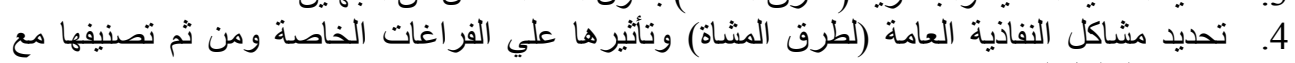

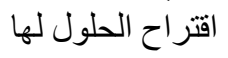

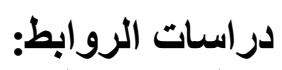

وتتعلق بشوارع السيار ات العامة والخدمة علي مستوي المدينة والبيئة المحيطة المجاورة و القريبة و علاقاتها بطرق 1

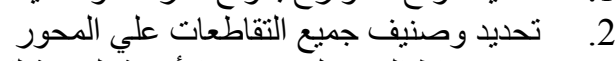

3. تحديد نقاط الوصول وتحديد الأهمية النسبية لكل منها (شكل رقم 2)

4. تحليّل حركةَ مرورِ كُلّ الثوارع وفحص أعراض تللك الشوارع وتقاطعاتها وتحديد كثافة الاستعمال بكل

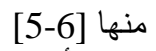
5. توقيع أماكن الطرقَ الجديدةً في الموقع - إذا أظهرت الدراسة الحاجة إليها- ومن ثم تصميم تقاطعاتها مع

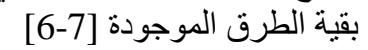

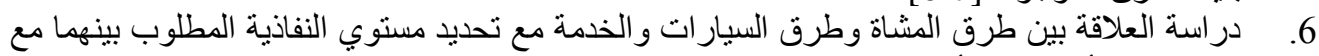
الحفاظ علي أعلي نسبة أمان مطلوبة العنة 


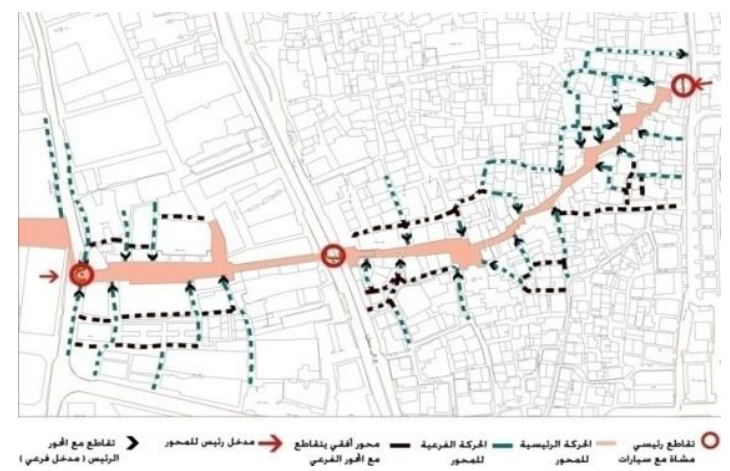

شكل رقم 2: مثال من لوحات الطلاب المقدمة للتقاطعات ونقاط الوصول و الحركة بمحور العلوي[8]

2.3

المفهوم : لغويا يعني التتوع التعدد الذي يحمل معني الاختلاف ويتضمن التتوع هنا في هذه الدراسة كافة المناطق

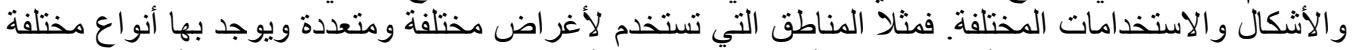

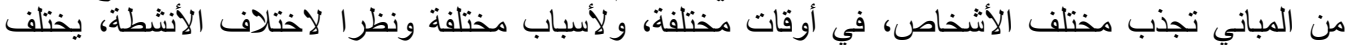

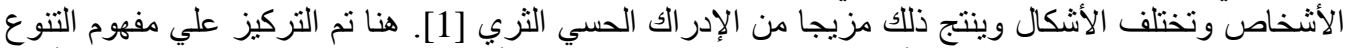

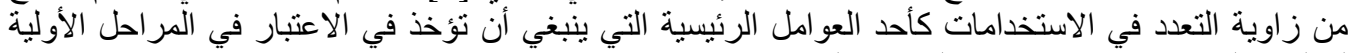

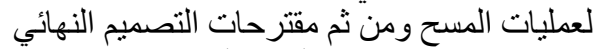

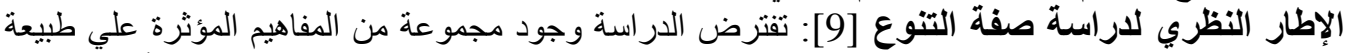

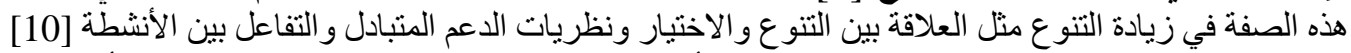

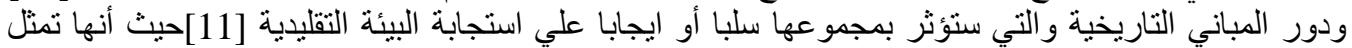

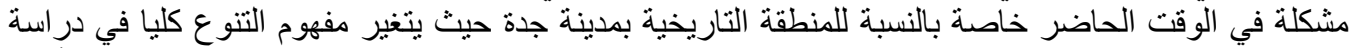

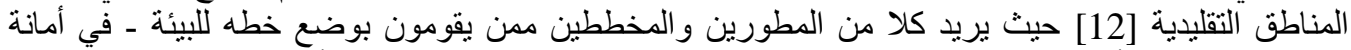

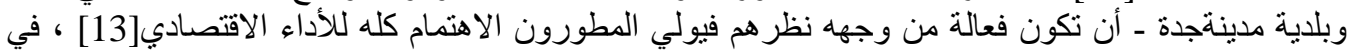
حين برغب المخططون في وضع المناطق المناسبة لإدارتها و للوصول إلهون إليها بكل سهوله [114].

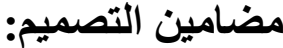

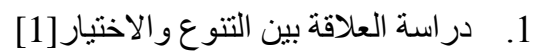
2.

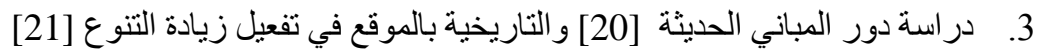

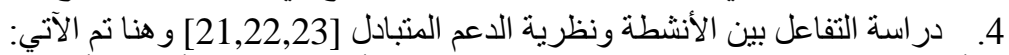

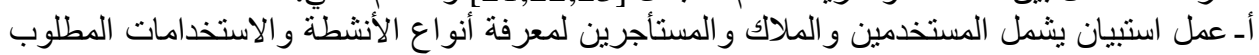
استحداثها والأشطة التي ينبغي المحافظة عليها أو تغيير أماكنها بـ تحديد المواقع الميزة ومواقع جذب الأشخاص و المشاة الرئيسية والثانوية ودراسة الدعم المتبادل فيما

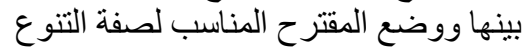

جـ-تحديد الاستخدامات المتقبية واقتراح سبل تقليل وتحجيم التفاعلات السلبية فيما بينها

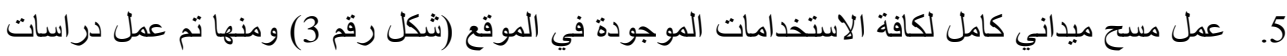
عامل الزمن و الجدوى الوظيفية ( دون التركيز علي الجدوى الاقتصادية والجدوى السياسية) [24] 


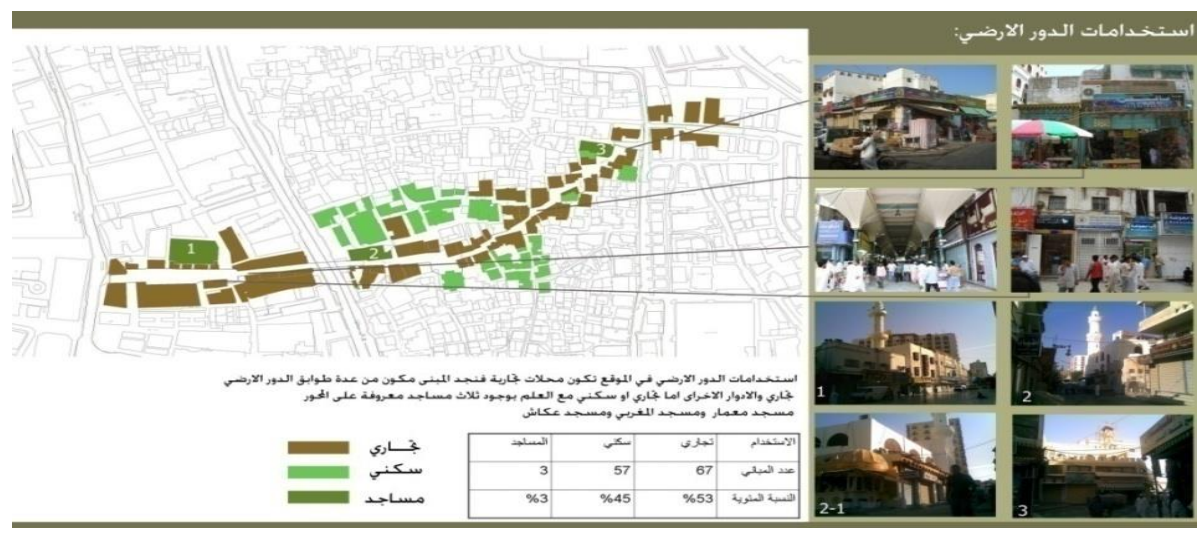

شكل رقم 3: مثال من لوحات الطلاب المقدمة لاستخدامات الدور الأرضي في محور العلوي [8]

3.3 الوضوح

المفهوم: الوضوح هو الخاصية التي تَجْعل المكان قابلا للإدر اكَ الحسي [1]

\section{الاطار النظري لاراسة صفة الوضوح [25]}

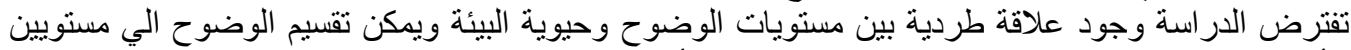

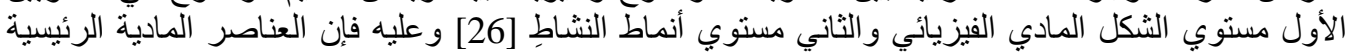

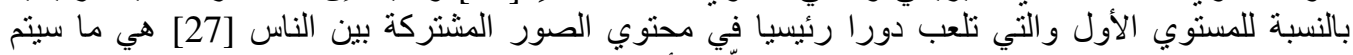

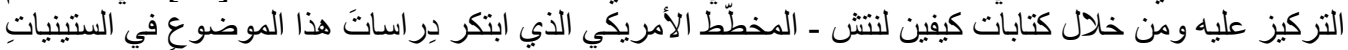

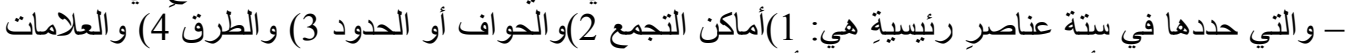

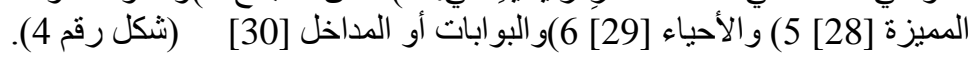

\section{مضامين التصميم:}

لتحقيق الوضوح في محور الدر اسة نتبع الخطوات بالترتيب التالي:

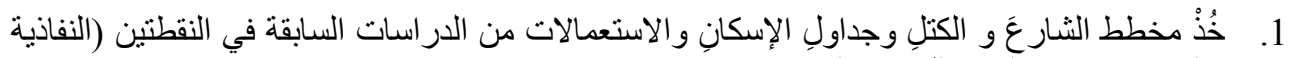

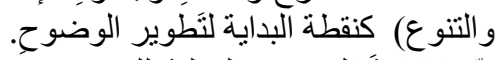

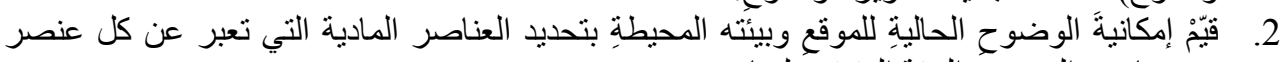

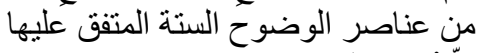

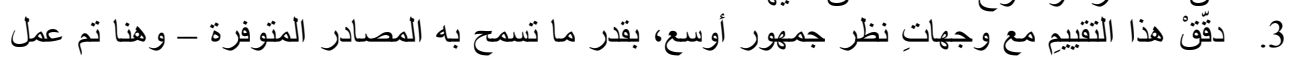

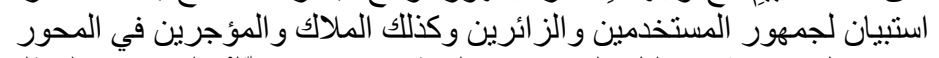

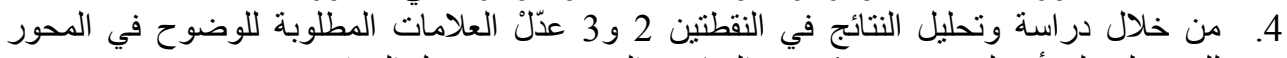

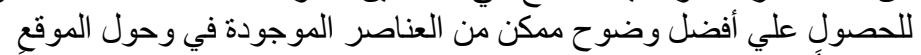

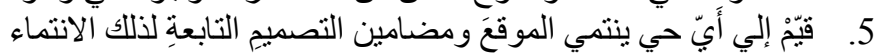

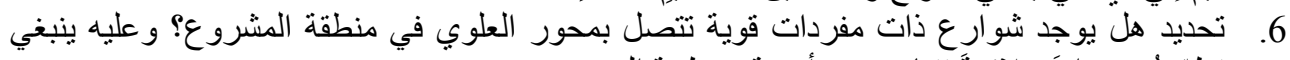

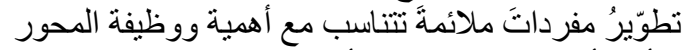
7.

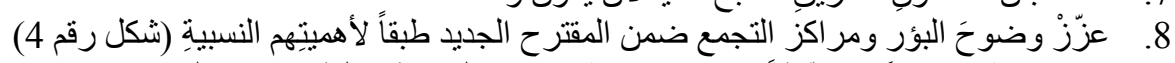

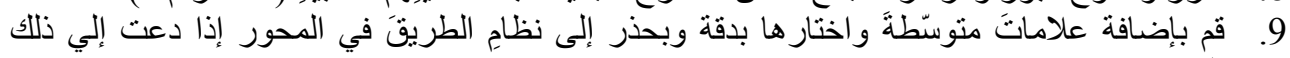

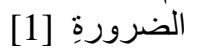



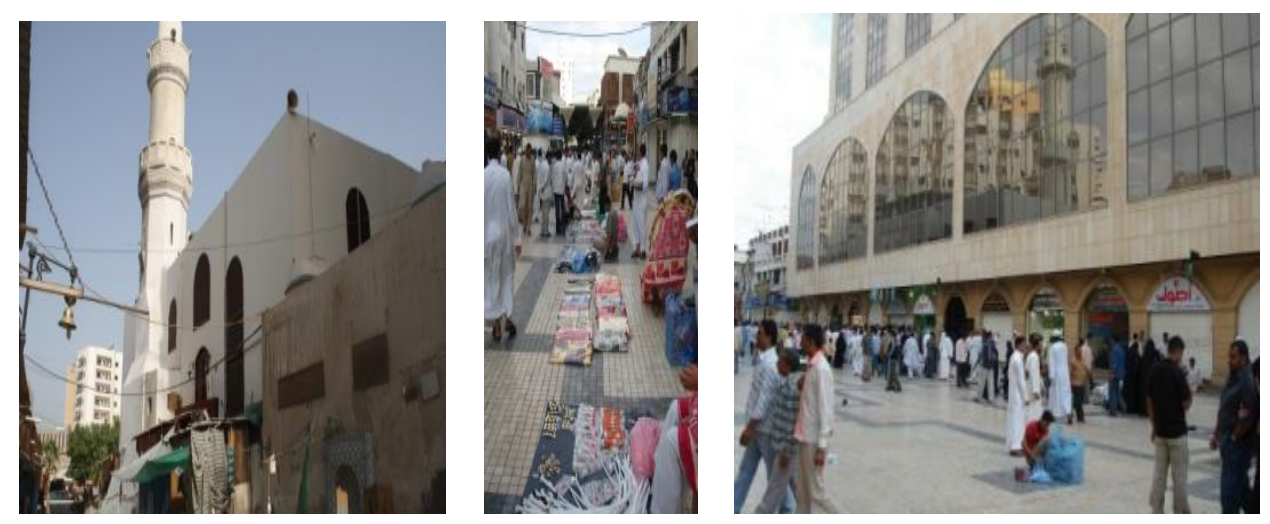

شكل رقم 4: يوضح عناصر الوضوح المادية (المعالم وأماكن التجمع والحدود والطريق) [31]

\section{3}

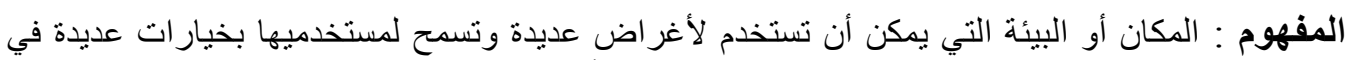

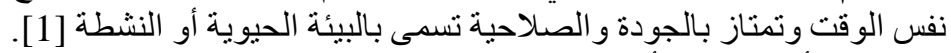

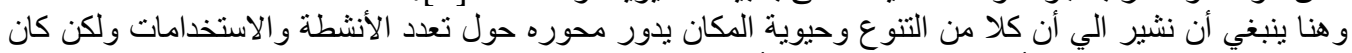

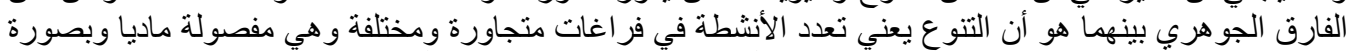

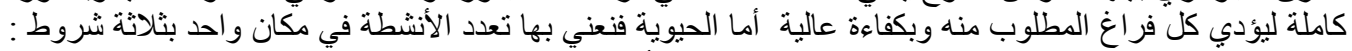

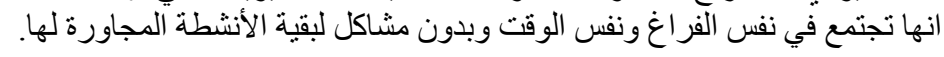

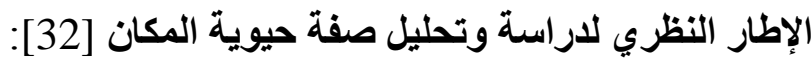

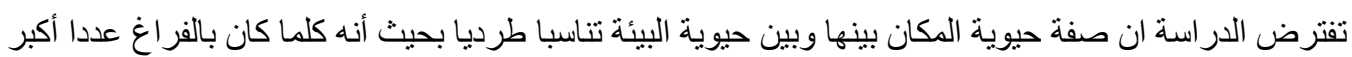

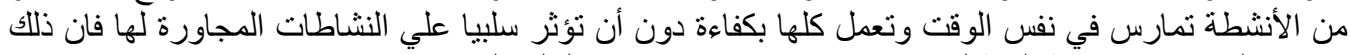

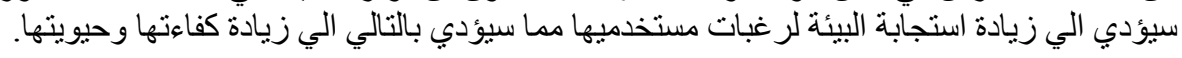

\section{مضامين التصميم}

1. يتم تقسيم العمل إلى مقياس كبير ومقياس صغير [33] [35. المقياس الكبير يهتم بالفراغات الكبيرة

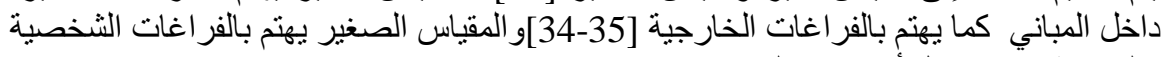

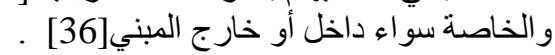

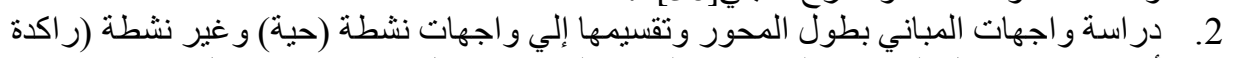

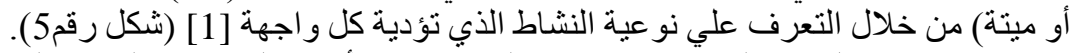

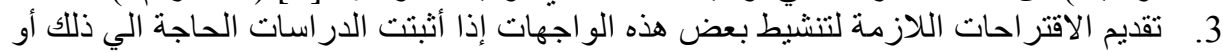

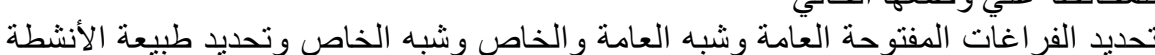

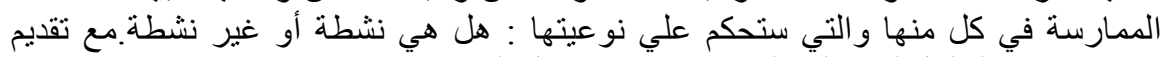

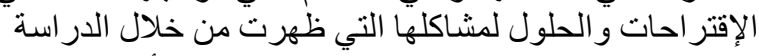

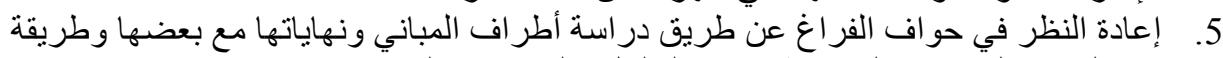

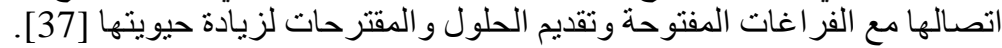

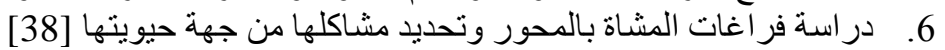

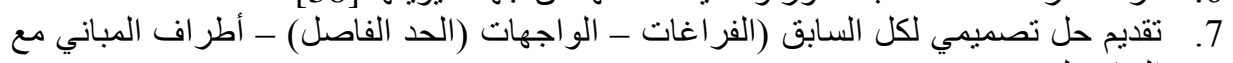

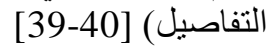
8. در اسة الرياح في المنطقة وتأثير ها علي معطيات التصميم [41] 


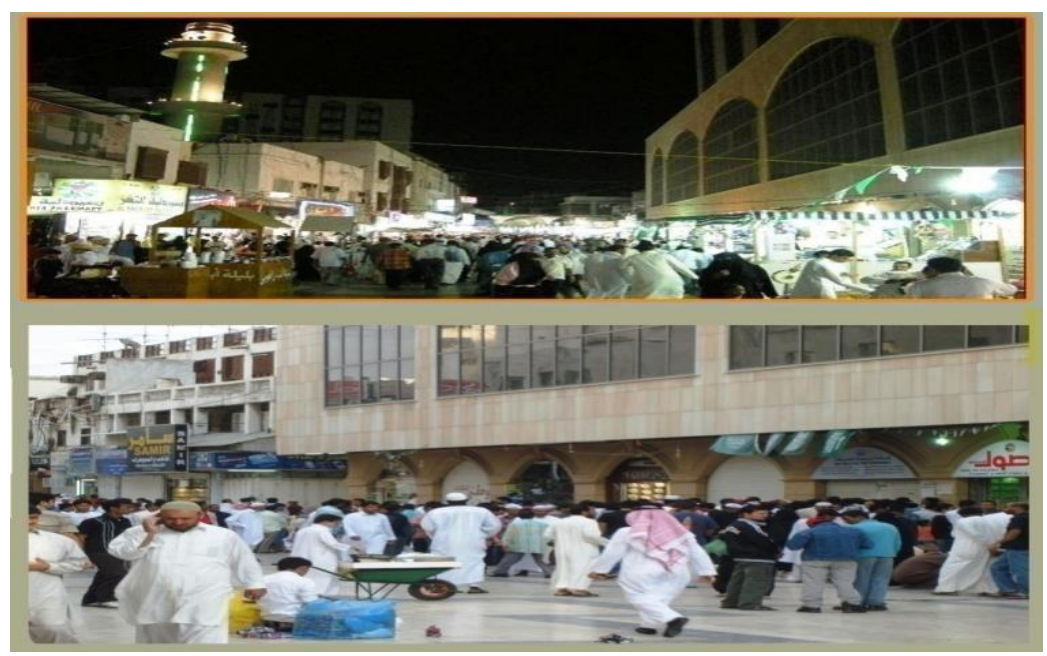

شكل رقم 5: مثال علي الساحات النشطة [31]

\section{3}

المفهوم: هي المظهر التفصيلي للمكان والذي يؤثر على ترجمة الناس لهذا المكان [42] و عندما تدعم هذه

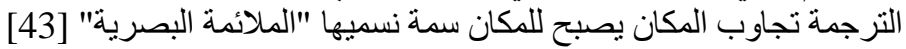

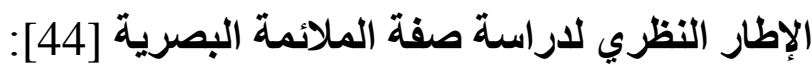

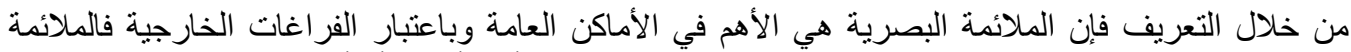

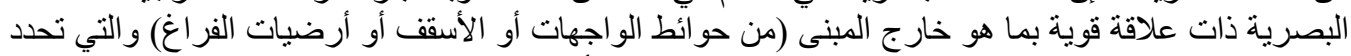

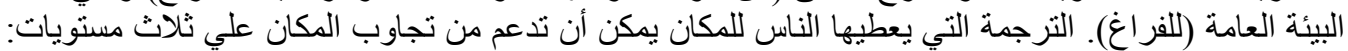

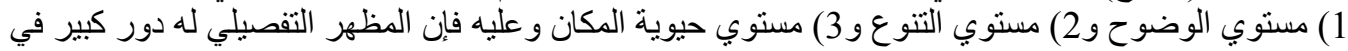

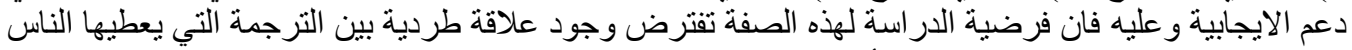

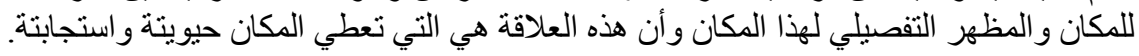

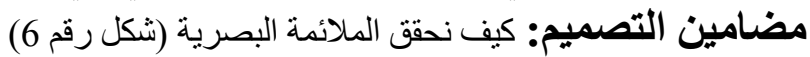

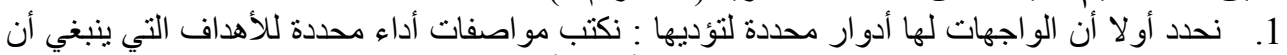

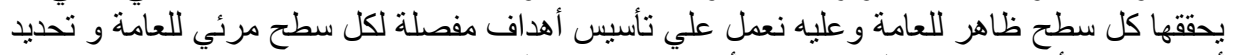

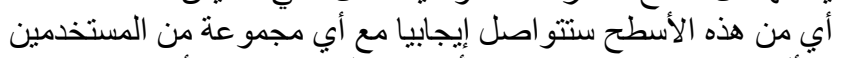

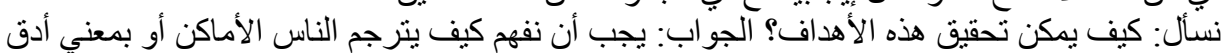

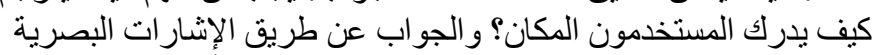

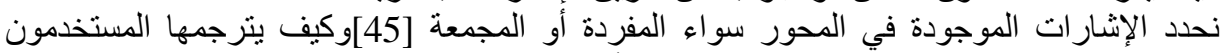
كعلامات لها معاني تختلف بأختلاف التجارب والأ والأهداف لكل منهم (وهنا يتم عمل استبيان لمستخدمي

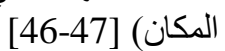

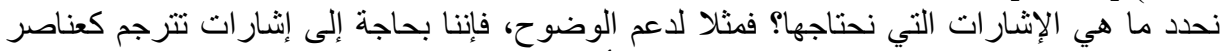

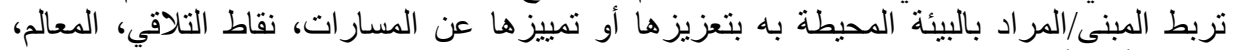

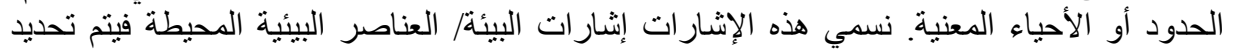

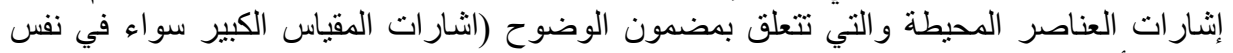

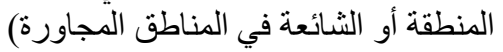

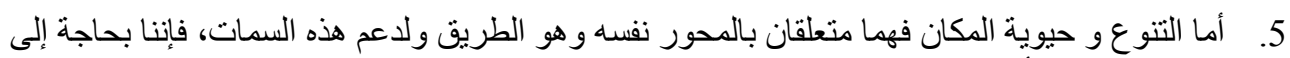

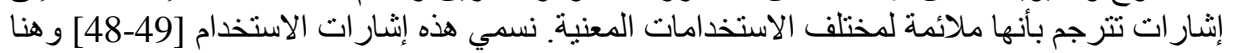


يتم تحديد إثارات عناصر الاستخدام والتي تتعلق بكل من مضمون التنوع ومضمون حيوية المكان

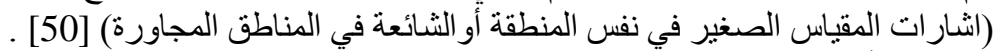

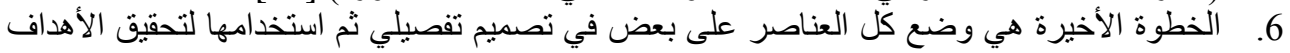

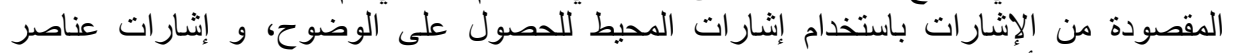

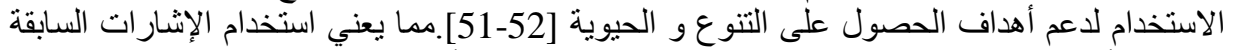

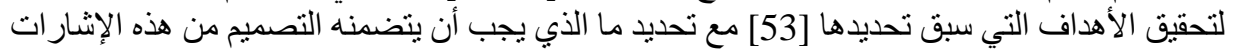

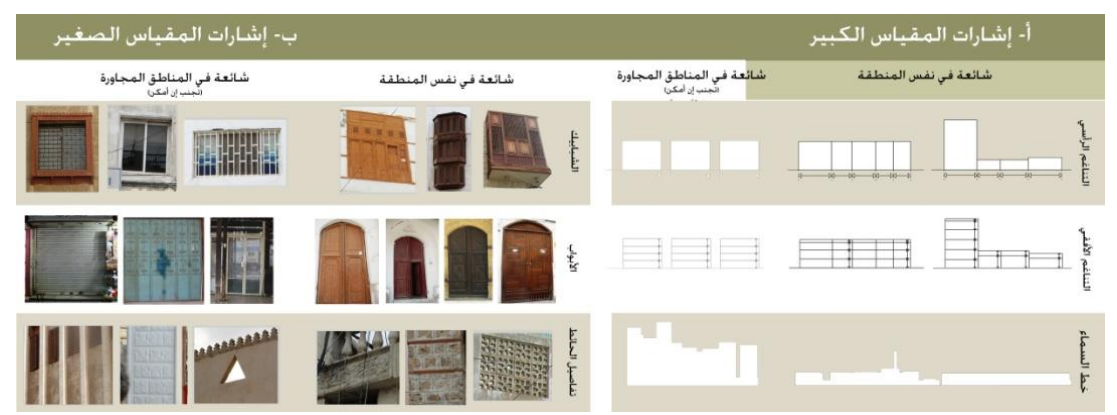

شكل رقم 6: نموذج من أعمال الطلاب يبين الاشار ات البصرية في محور العلوي [8]

\section{3 صفة الغني أو شراء الجودة}

المفهوم: ونعني به الأساليب والطرق التي تزيد من تنوع الخبرات التي يمكن أن تكتسب عن طريق التبات الحواس

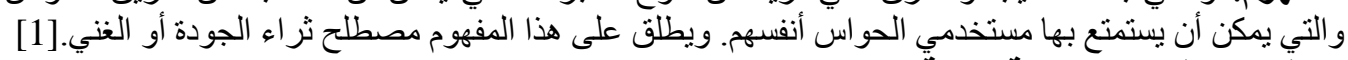

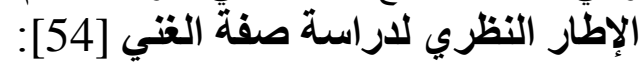

تعتبر حاسة البصر هي الحاسة المسيطرة لمعظم الناس. ومعظم المعلومات التي نتعامل معها تأتينا من خلال أعبات أعينا

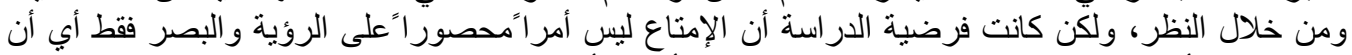

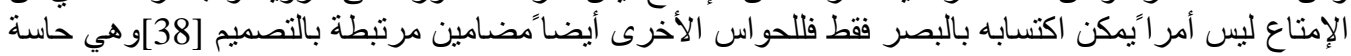

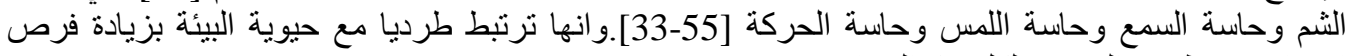
الامتاع عن طريق التصميم لكل هذه الحواس.

\section{مضامين التصميم:}

1. كان لابد لنا من السؤال: كيف يستطيع المستخدمون للمكان أن يختاروا ما يثري حو اسهم بجمال وروعة

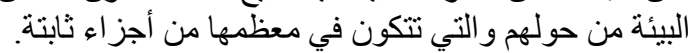

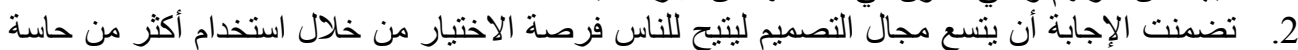

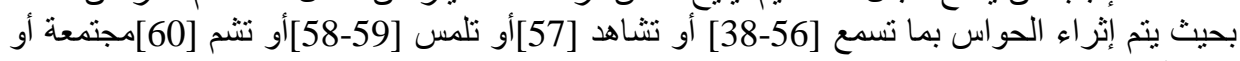
متفرقة هذبة لتحقيق هذا المفهوم من الإمتاع في التصميم كان علينا أن نهتم في المقام الأول بالأجزاء الصغيرة الثابتة

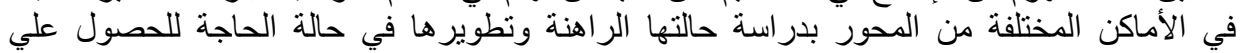

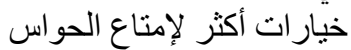
وضع أساسيات المتعة البصرية: حيث الإعت تعتمد المتعة البصرية على وجود الصور البصرية المتغايرة

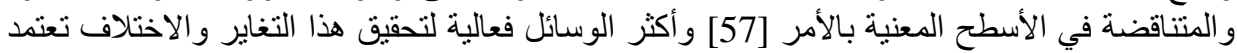
على عاملين رئيسيين هما: ba

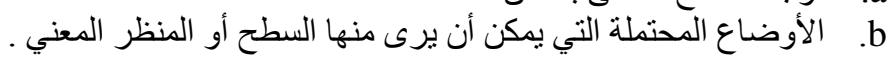




\section{أولا: الثراء البصري: - ماء}

1- خذ التصميم المفصل من الصفة الخامسة كأساس لتطوير إمتاع بصري إلمرئ إضافي .

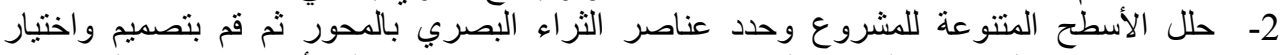

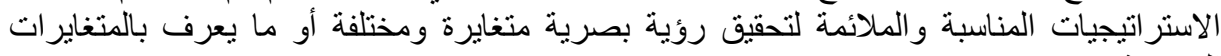
البصرية الانئة 3- حلل مسافات الرؤية البصرية المحتملة والزمن المستغرق في الرؤية البصرية لكل سطح أو منظر

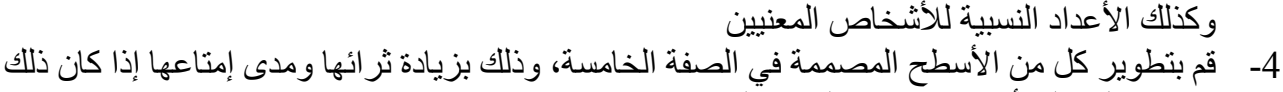

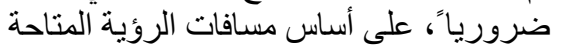

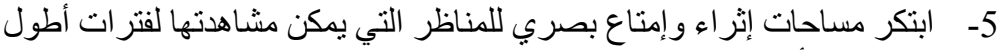

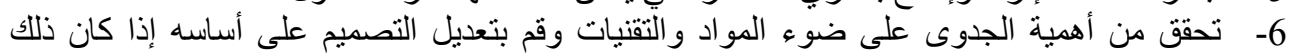
التعديل ضروريا ولا بد منه الجنه.

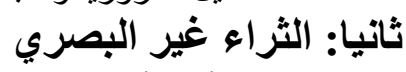

7- رر أي الأماكن يمكن أن لا يكون فيها مصدر إمتاع بصري، والهري واعمل تصميمك على أساس مستمد من

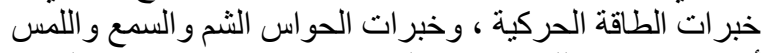

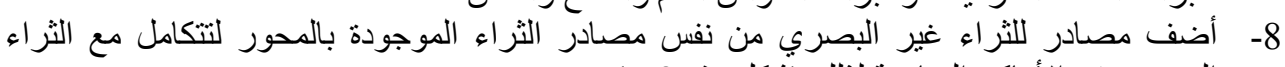
البصري في الأماكن المناسبة لذلك (شكل رقم
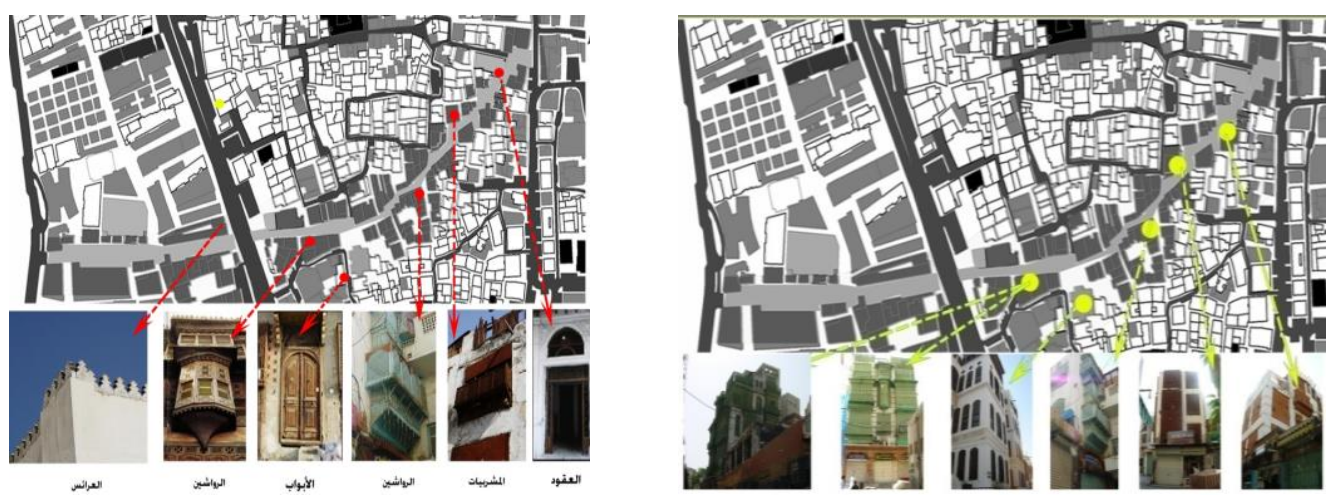

شكل رقم 7: الثراء البصري في محور العلوي [8]

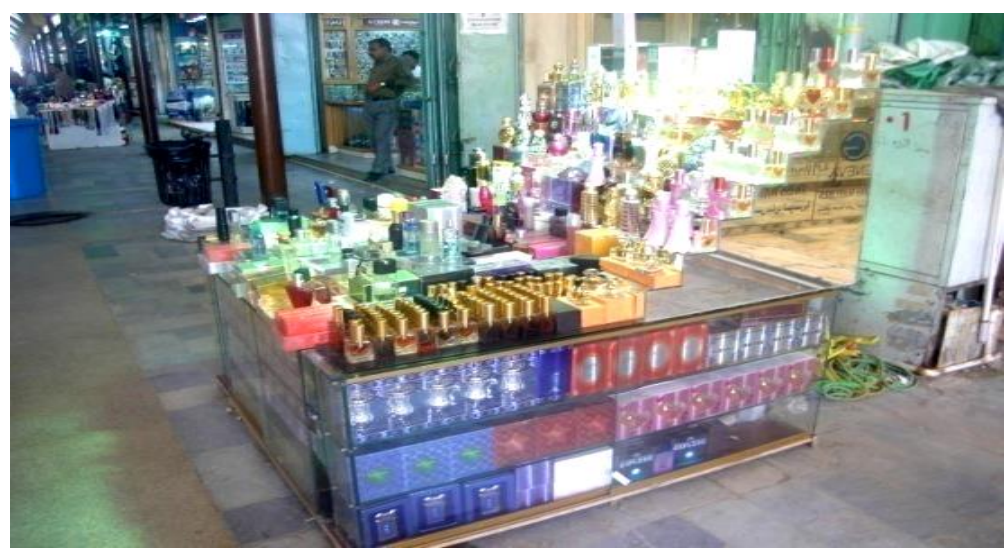

شكل رقم 8: بسطات الروائح المتعددة تحت المظلات هي أحد مصادر الثراء و الامتاع (الثمي) غير البصري في

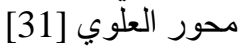




\section{3}

المفهوم : هو الصفة التي بيتطيع غالبية الأشخاص من خلالها تحقيق بيئة ذات طابع بحمل أذواقهم وقيمهر

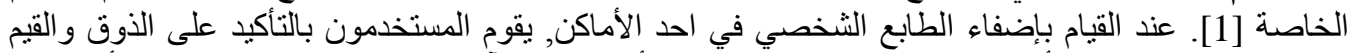

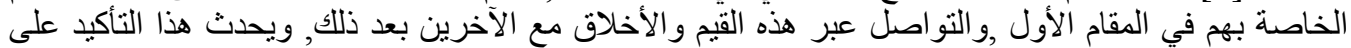

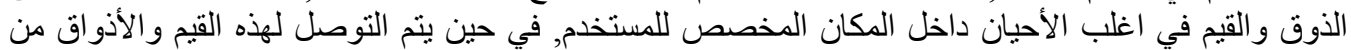

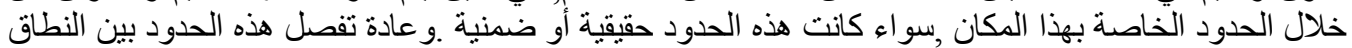

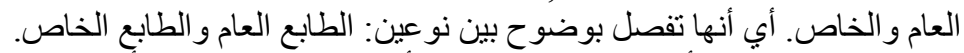

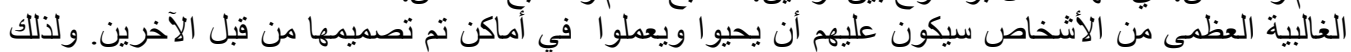

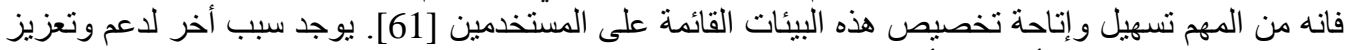

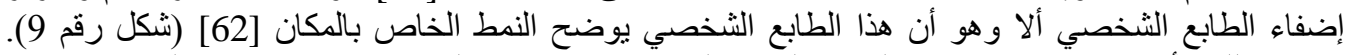

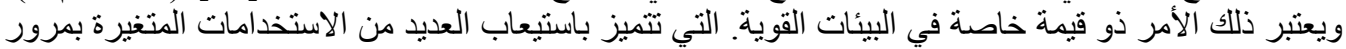

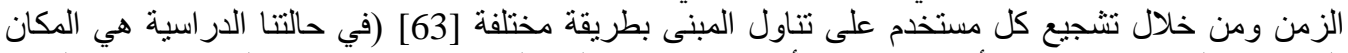

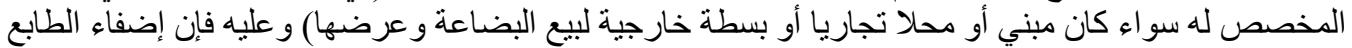

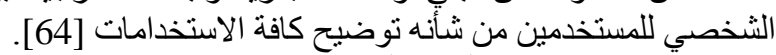
الإطار النظري لاراسة صفة إعطاء الطابع الشخصي [65]

ارنكز الإطار النظري لدر اسة صفة إضفاء الطابع الثخصي علي أن البيئة التي تحمل قيم و أذواق مستخديها تزيد

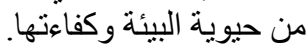

\section{مضامين التصميم}

1. تحديد طرق إضفاء الطابع الثخصي (التأكيدي و العلاجي) و الفرق بينهما ومني نستخدمهما

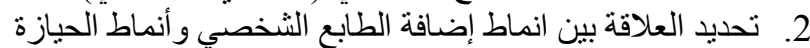

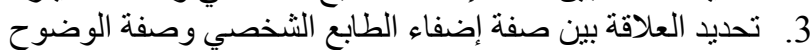

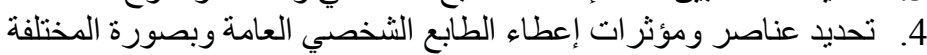

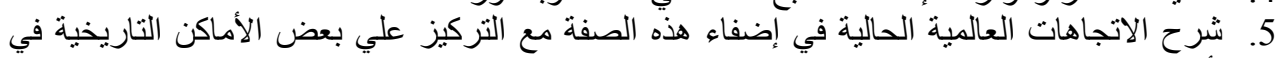

أماكن مختلفة (مصر - شارع المعز لدئ لدين الله كمثال)

6. تحديد أين ينم تطبيق التشخيص؟

7. بعد الجزء النظري السابق يتم اخذ التصميم من الصفة السادة كأحد النقاط الأولى لبدء الششروع

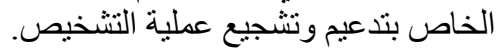

8. ثم يتم تطوير التصميم التفصيلي للأوجه الداخلية (عند الاستطاعة بوجود الخرائط وامكانبة الدخول

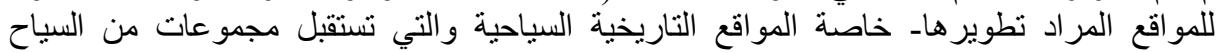
و والزوار (بيت نصيف كمثال)

9. يتم تطوير المخططات التصميمية للمداخل (العتبات) الداخلية والخارجية

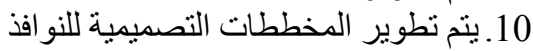

11. ينت تطوير المخططات التفصيلية للأوجه الخارجية, ثم يتم تقييم التأثيرات الدحتملة للتشخيص المرئي العام ومن ثم يتم تعديل التصميم عند الضرورة 


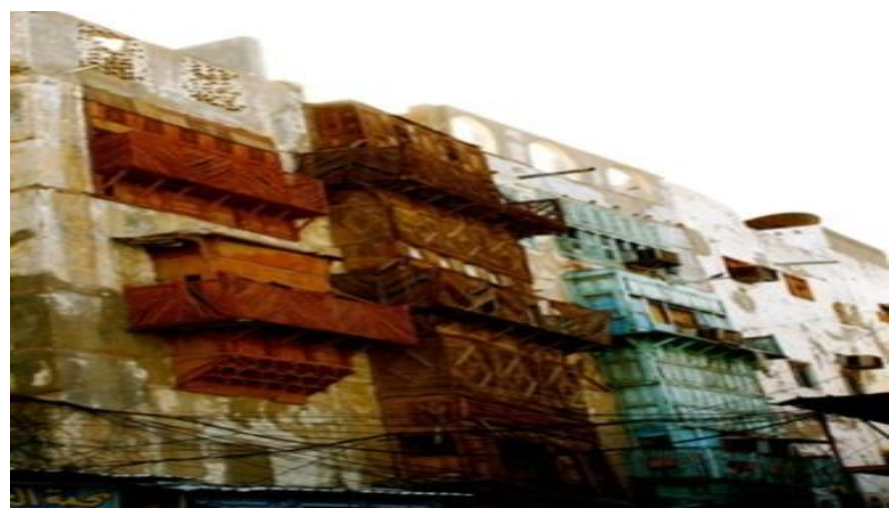

شكل رقم 9: بعض عناصر اضفاء الطابع الشخصي كما تظهر في محور العلوي[31]

4

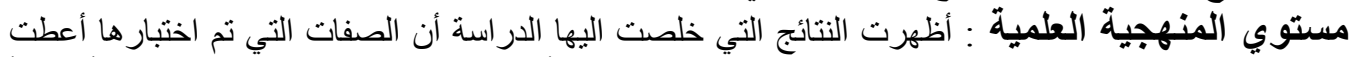

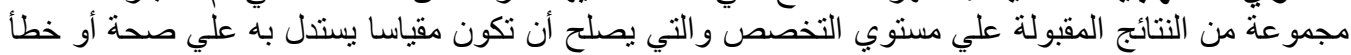

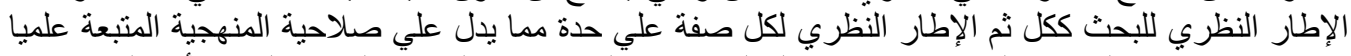

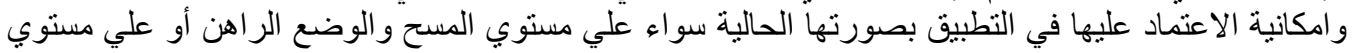

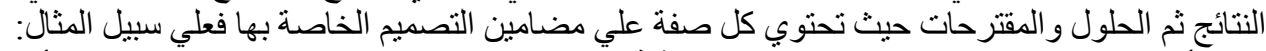

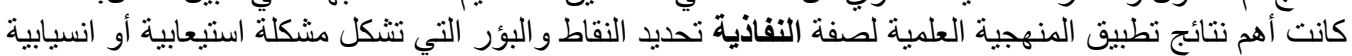

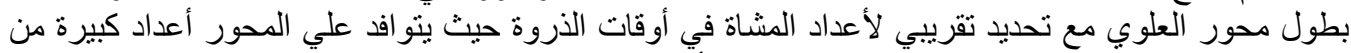

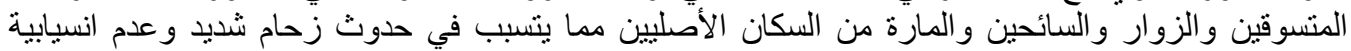

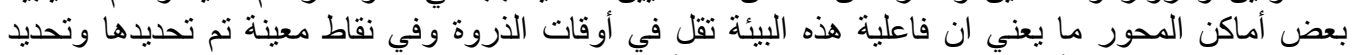

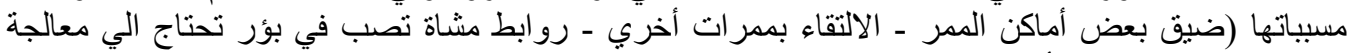

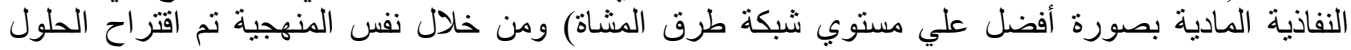

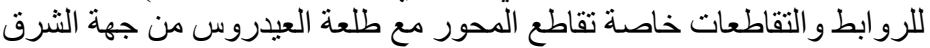

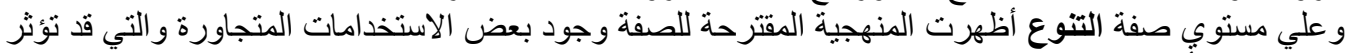

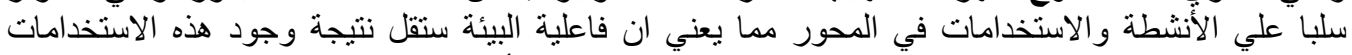

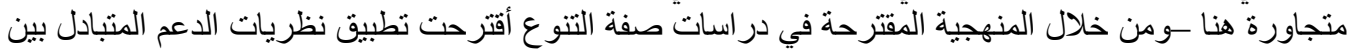

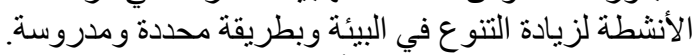

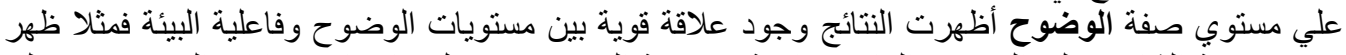

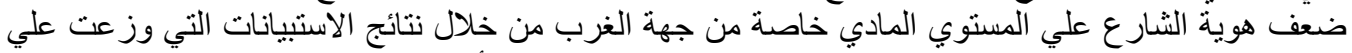

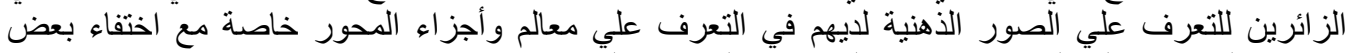

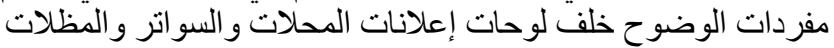

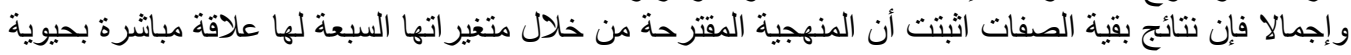

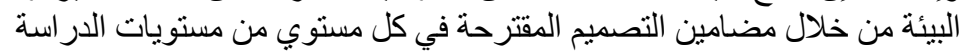

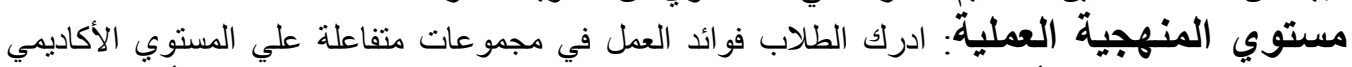

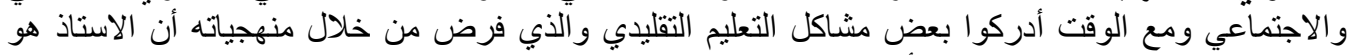

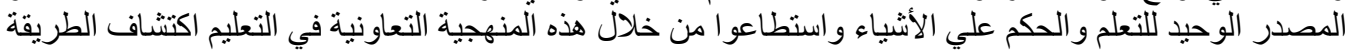

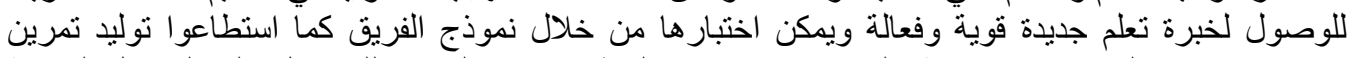

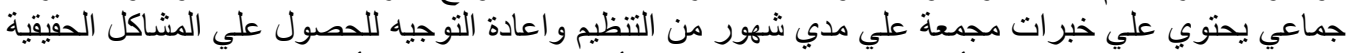

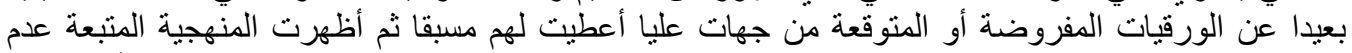
مصداقية كثيرمن مشاكل الموقع محل هذه الدراسة من خلاتل هذا التمرين فاستخدم الطلاب التقييم كأداة للحكم و التعليم في نفس الوقت مثن 
و اثتتت المنهجية فاعليتها كذللك من جهة نوزيع المهام علي الطلاب بهذه الطريقة مع إعطاء كل منهم مسئولية صفة

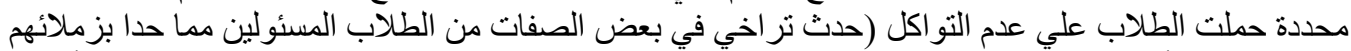

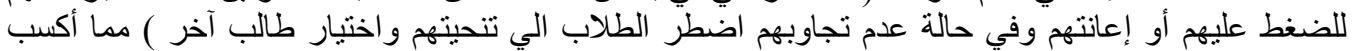

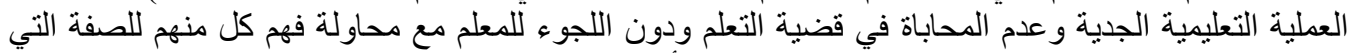

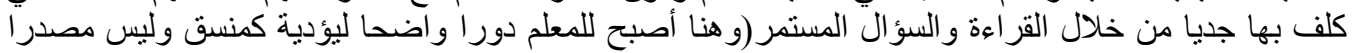

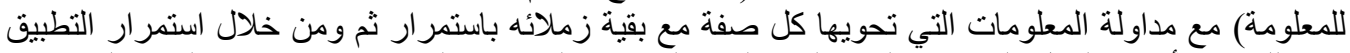

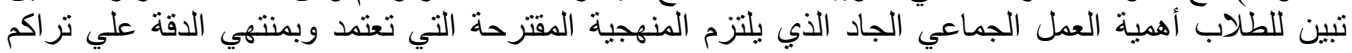

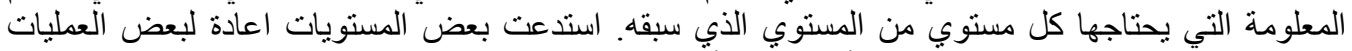

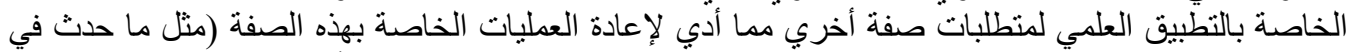

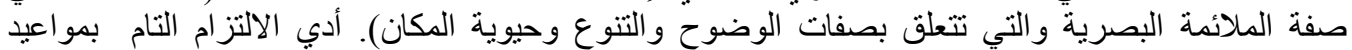

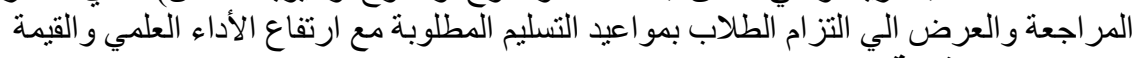

5. المناقشة والتوصيات الترات

قدم هذا البحث إضافات علمية وعملية فمن الناحية العلمية فإن دراسات التصميم الحضري من خلان خلال تطبيق

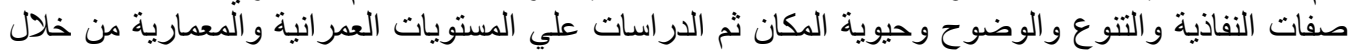

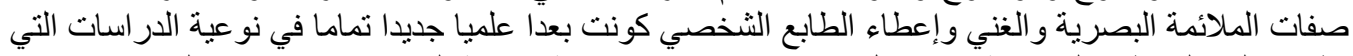

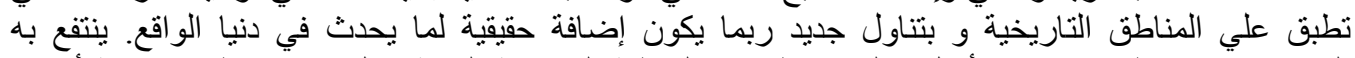

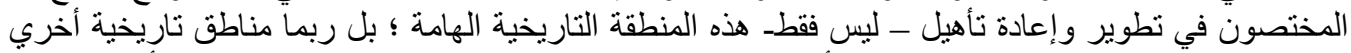

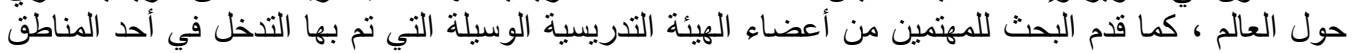

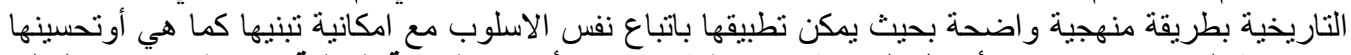

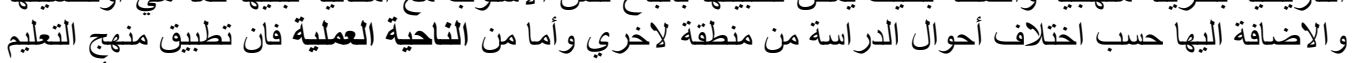

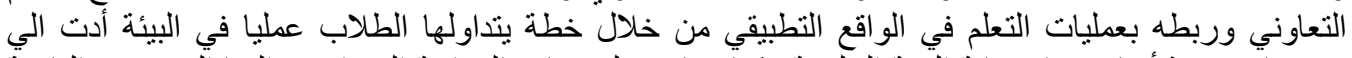

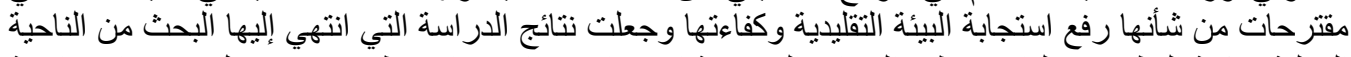

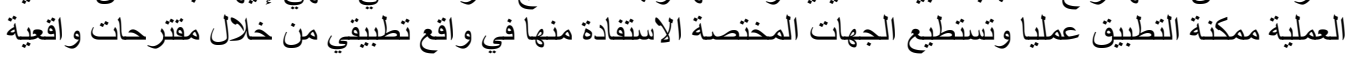
تم التوصل إليها علي المستويين العمر اني و المعماري.

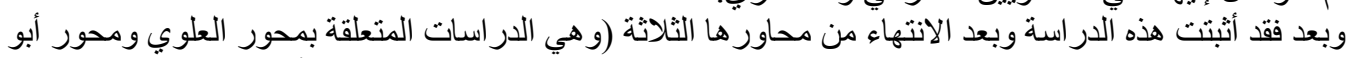

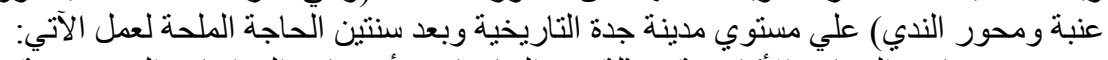

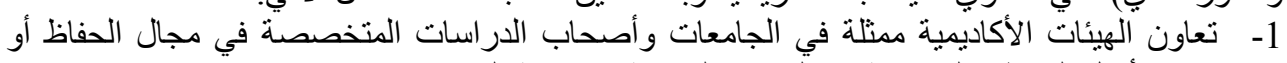

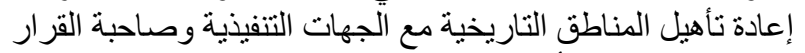

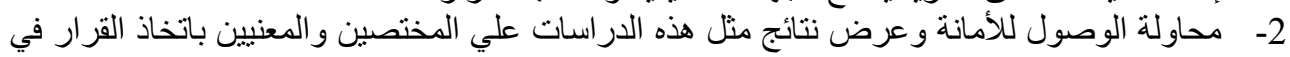
المنطقة التاريخية .

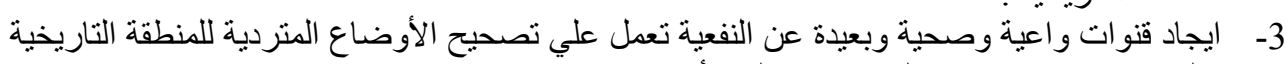
و التي ربما تتكرر وبنفس الصورة فئورة في بلدان أخري

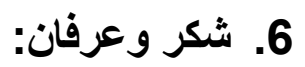

يشكر الباحث الاخوة الزملاء الذين تفضلوا بمر اجعة البحث و ابداء بعض الماتلاحظات القيمة التي أوصلت الدراسة

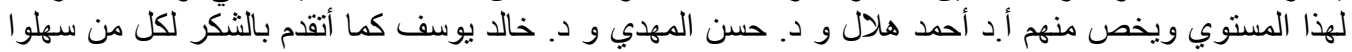

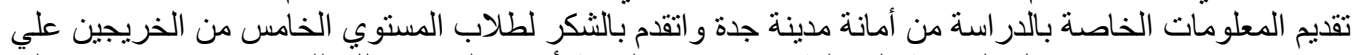

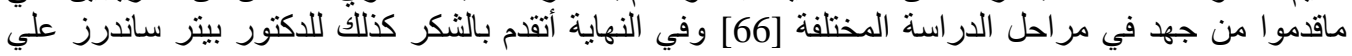

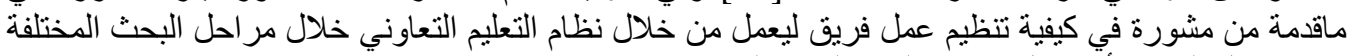

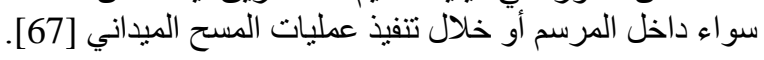




\section{المراجع}

[1] Bentley, I, (1998) Responsive Environments: a manual for designers, Architectural Press, Linacre house, Jordox Hill, Oxford OX2 8D.

[2] Jeddah Municipality (2006), Master Action Plan for Jeddah - final report.

[3] Peter M, Saunders, Introduction to Collaborative Learning Teams, Center for teaching and learning Development, King Abdulaziz University.

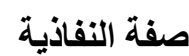

[4] Kassar, N, (2009) Permeability and the Nature of Historical Areas, a series of lectures, Video archive of Human Architecture Unit, Department of Architecture, King Abdul-Aziz University.

[5] Department of the Environment and Department of Transport (1977) Design Bulletin 32, London: DoE and DoT.

[6] Department of Transport (1966) Roads in urban areas, London: DoT.

[7] Noble, J. (1983) Local standards for the layout of residential roads: a review, London: Housing Research Foundation.

[8] Students Final Presentation (2009), Studio archive - Human Architecture Unit, Department of Architecture, King Abdul-Aziz University.

[9] Kassar, N, (2009) Concept of Variety in Historical Areas, a series of lectures, Video archive of Human Architecture Unit, Department of Architecture, King Abdul-Aziz University.

[10] Bates, J. et. al. (1978) Research report 20: A disaggregate model of household car ownership, London: Department of Transportation

[11] Bates, J. et. al. (1981) The factors affecting household car ownership, Farnborough: Gower.

[12] Bentley, I. (1983a) Bureaucratic patronage and local Urban form, (JCUD Research Note 15) Oxford: Joint Centre for Urban Design, Oxford Polytechnic.

[13] Municipality of Jeddah City, (2009), "Towards First World Class" Workshop for Jeddah Central Area, Minisry of native and village affairs, Kingdom of Saudi Arabia.

[14] Municipality of Jeddah City, (2007) King Abdul Aziz Projcet for Protection and development of Jeddah Historical Area, Minisry of native and village affairs, Kingdom of Saudi Arabia.

[15] Questionnaire, (2009) Human Architecture Unit, student survey.

[16] Barrett, J. (1979) The form and functions of the central area, (Open University DT 201 Unit 12), Milton Keynes: Open University.

[17] Markus, T. (ed) (1979) Building conversion and rehabilitation: designing for change in building use, London: Butterworth.

[18] Bowyer, J. (1979) Guide to domestic building surveys, London: Architectural Press.

[19] Bathurst, P.E. and Butler, D.A. (1980) Building cost control techniques and economics, London: Heine-mann.

[20] Jacobs, J (1965) The death and life of great American cities, Harmondsworth: Penguin. 
[21] Tutt, P and Adler, D. (eds) (1981) New Metric Handbook, London: Architectural Press.

[22] Williams, B and associates (1976) Property development feasibility tables, London: Building Economics Bureau.

[23] Booth, R.D.B. (1984) Early perspectives in the valuation of property, Oxford: Elsfield.

[24] Heery, G. T.(1975) Time cost and architecture, New York: McGraw Hill.

[25] Kassar, N, (2009) The Notion of Legibility "a review for Kevin Lynch Book, the image of the city" a series of lectures, Video archive of Human Architecture Unit, Department of Architecture, King Abdul-Aziz University.

[26] Steinitz, C (1968) 'Meaning and congruence of urban form and activity', Journal of the American Institute of Planners, vol 34, pp233-247.

[27] Lynch, K. (1975) The image of the city, Cambridge (Mass):MIT Press.

[28] Ibid, chapter 3.

[29] Ibid, p169. Fig 56.

[30] Ibid, pp144 et seq.

[31] Special Collection, Old Jeddah, Courtsy of Kassar, Nabil, King Abdulaziz University.

[32] Kassar, N, (2009) Robustness, a Theoretical Framework, a series of lectures, Video archive of Human Architecture Unit, Department of Architecture, King Abdul-Aziz University.

[33] Whyet, W. H. (1980) The social life of small urban spaces, Washington D.C: Conservation Foundation.

[34] Alexander, C. (1971) Notes on the synthesis of form, Cambridge (Mass): Harvard University Press.

[35] Shills, E. (1981) Tradition, London: Faber and Faber.

[36] Cowan, P. (1963) 'studies in the growth, change and ageing of buildings in Transactions of the Bartlett Society, Vol VI.

[37] Appleton, J. (1975) The experience of landscape, London (etc): Wiley.

[38] Project for Public Spaces Inc. (1982) Effective pedestrian improvements in downtown business districts, (Planning Advisory Service Report No. 368) New York: American Planning Association.

[39] Grotenhuis, D. H. (1978) The woonerf in city and traffic planning, Delft, Netherlands: Traffic Department, Public Works Services, Municipality of Delft.

[40] Royal Dutch Touring Club (1980) Woonerf, (2nd .ed.), The Hague: RDTC Traffic department.

[41] Penwarden, A.D. and Wise, A.F.E. (1975) Wind environment around buildings: a Building Research Establishment report, London: HMSO.

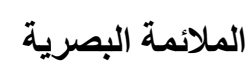

[42] Bonta, J.P. (1979) Architecture and its interpretation, London: Lund Humphries.

[43] Broadbent, G. (1977) 'A plain man's guide to the theory of signs in architecture' in Architectural Design, 1977, 7-8 pp 474-482. 
[44] Kassar, N, (2009) Visual Appropriateness and Responsive Environments, a series of lectures, Video archive of Human Architecture Unit, Department of Architecture, King Abdul-Aziz University.

[45] Bernstein, B. (1971) Class, codes and control, (3 vols) London: Routledge and Kegan Paul.

[46 ]Bourdieu, P. (1980) 'The aristocracy of culture' in Media, Culture and Society, 1980, 2, pp 225-254.

[47]Clarke, L. (1973) Explorations into the nature of environmental codes: the relevance of Bernstein's 'theory of Codes' to environmental Studies, Working Paper No. 8 September 1973. Kingston: Architectural Psychology Research Unit, Kingston Polytechnic School of Architecture.

[48] Douglas, M. (1978) Cultural bias, Royal Anthropological Institute, Occasional Paper No. 35.

[49] Douglas, M. (1982) Essays in the sociology of perception, London: Routledge and Kegan Paul.

[50] Appleyard, D. (1969) 'Why buildings are known: a predictive tool for architects and planners' in Broadbent, G. et. al. (eds) (1980) Meaning and behavior in the built environment. Chichester: Wiley.

[51] Berger, P. and Luckmann, T. (1966) The social construction of reality, Harmondsworth: Penguin.

[52] Moore, G.T. (1983) 'Knowing about environmental knowing: the current state of theory and research on environmental cognition' in Pipkin, J.S. et. al. (eds) (1983) Remarking the city: social perspectives on urban design, New York: State University of New York Press

[53] Hanson, J and Hillier, B. (1982) 'Domestic space organization: two contemporary space-codes compared' in Architecture and Behavior 1982, 2, pp. 5-25.

$$
\text { ثراء الجودة - مالغني }
$$

[54] Kassar, N, (2009) Richness, Design for All Senses, a series of lectures, Video archive of Human Architecture Unit, Department of Architecture, King Abdul-Aziz University.

[55] Gibson, J. J. (1966) The senses considered as perceptual systems, Boston: Houghton Mifflin.

[56] Filler, M. (1978) 'Extra sensory perceptions' in Progressive Architecture. Vol 59 No. 4, April 1978, pp. 82-85.

[57] Miller, G. (1956) ' The magical number seven, plus or minus two: some limits on our capacity for processing information' in the Psychological Review, vol 63, March 1956.

[58] Halprin, L. (1969) The RSVP cycles: Creative processes in the human environment, New York: Braziller.

[59] Process Architecture (1978) Lawrence Halprin, Special issue, February 1978.

[60] Garland, S. (1984) The herb garden, Leicester: Wind-ward.

[61]Bentley, I. (1983b) 'Designing responsive places' pp. 8-11, in Urban Design Quarterly, London: Urban Design Group. 
[62] Grimsby Borough Council (1976) House improvements and the street scene, Grimsby: Borough Council.

[63] Wolverhampton Borough Council (1978) Streets ahead: a guide to improving the appearance of your house, Wolverhampton: Borough Council.

[64] Hertzberger, H. ' Looking for the beach under the pavement ' in RIBA Journal Vol 78. August 1971, pp. 328-333.

[65] Kassar, N, (2009) Personalization, Planning Debates about Control Versus Individual Choice, a series of lectures, Video archive of Human Architecture Unit, Department of Architecture, King Abdul-Aziz University.

[66] Students of 5th year (2009): Mohamed Bin Shayhoon, Saleh Bin Shayhoon, Mishal Al Maimani, Saleh Al Sahali, Ahmed Al Jehani and Mohammad Nas, Department of Architecture, Faculty of Enviromenal Design, King Abdul Aziz University.

[67] Saunders, M, Peter, Director (2009), Oregon State University, Center for Teaching and Learning Effectiveness. 